\title{
Overexpression of Sirt6 promotes M2 macrophage transformation, alleviating renal injury in diabetic nephropathy
}

\author{
LIQIANG JI ${ }^{1}$, YIFANG CHEN ${ }^{1}$, HONGQIANG WANG ${ }^{2}$, WEI ZHANG ${ }^{1}$, \\ LANXIANG HE ${ }^{1}$, JINGMIN WU ${ }^{1}$ and YINGHUI LIU ${ }^{3}$ \\ ${ }^{1}$ Department of Pharmacy, The First People's Hospital of Yuhang District, Hangzhou, Zhejiang 311100; \\ ${ }^{2}$ Department of Pharmacy, Hangzhou Yuhang TCM Hospital, Hangzhou, Zhejiang 311106; \\ ${ }^{3}$ Department of Endocrinology, Zhejiang Provincial Integrated Chinese and Western Medicine Hospital, \\ Hangzhou Red Cross Hospital, Hangzhou, Zhejiang 310003, P.R China
}

Received October 9, 2018; Accepted March 1, 2019

DOI: 10.3892/ijo.2019.4800

\begin{abstract}
In this study, we aimed to investigate the associations between Sirt6, macrophages and diabetic nephropathy (DN). Immunohistochemical, western blot and RT-qPCR analyses were performed to detect the expression levels of Sirt6, the markers of podocytes and monocytes and related inflammatory factors in the tissues of rats with streptozocin-induced DN. A series of cell experiments in isolated culture or the co-culture of macrophages and podocytes were conducted to examine the effects of the overexpression of Sirt6 on macrophage transformation, podocyte apoptosis and associated genes, and analyses were performed using RT-qPCR, flow cytometry and western blot analysis, where appropriate. In the rat model of DN, injured podocytes were represented by the decreased protein expression levels of Nephrin and Sirt6, and by an increased Desmin expression. Additionally, the M1 phenotype transformation of macrophages was evidenced by the increased expression levels of CD86, tumor necrosis factor (TNF)- $\alpha$ and inducible nitric oxide synthase (iNOS), and by the decreased expression levels of CD206, Sirt6, interleukin (IL)-4 and IL-10. In vitro assays of macrophages and podocytes demonstrated that glucose promoted macrophage M1 transformation and podocyte apoptosis in a dose-dependent manner and attenuated Sirt6 expression. Macrophages transformed into the M2 phenotype following the overexpression of Sirt6 by the successful transfection of macrophages with a Sirt6 overexpression plasmid. Sirt6 was also overexpressed in podocytes. In a
\end{abstract}

Correspondence to: Dr Yinghui Liu, Department of Endocrinology, Zhejiang Provincial Integrated Chinese and Western Medicine Hospital, Hangzhou Red Cross Hospital, 208 Huancheng East Road, Hangzhou, Zhejiang 310003, P.R. China

E-mail: lyinghui_yhl@163.com

Key words: diabetic nephropathy, Sirt6, M1/M2 macrophages, podocyte
Transwell co-culture system, the overexpression of Sirt6 in macrophages (but not the overexpression of Sirt6 in podocytes) protected the podocytes from high-glucose-induced injury. However, the apoptosis of the podocytes overexpressing Sirt6 (induced by transfection with a Sirt6 overexpression plasmid) still increased when these podocytes were co-cultured with macrophages in high-glucose medium. These protective effects were evidenced by the inhibition of apoptosis, the upregulation of the expression levels of Bcl-2 and CD206, as well as by the decreased expression levels of Bax and CD86. On the whole, the findings of this study suggest that Sirt6 protects podocytes against injury in a mimicked diabetic kidney microenvironment by activating M2 macrophages, indicating that Sirt6 can act as an immune response regulatory factor in $\mathrm{DN}$-associated renal inflammatory injury.

\section{Introduction}

Diabetes mellitus (DM) is a global disease. According to the International Diabetes Federation, the incidence of diabetes is increasing worldwide, with an incidence of $5.4 \%$ in 2013 and 592 million cases occurring within 25 years (1). Approximately $80 \%$ of diabetes-related deaths occur in developing countries (1). Approximately $1 / 3$ of the patients with diabetes develop diabetic nephropathy (DN). As a type of microvascular disease, $\mathrm{DN}$ is one of the causes of mortality and disability to patients, and is also the main cause of end-stage renal disease (2). Glomerulosclerosis and tubular fibrosis are the main pathological characteristics of $\mathrm{DN}$, in which glomerular podocyte injury is an early event (3). As glomerular visceral epithelial cells, podocytes are highly differentiated renal parenchymal cells with various characteristics and functions, including endocytosis, the secretion of bioactive mediators and cytokines, and are involved in the local immune response of the kidneys (4-6).

The mononuclear macrophage system includes monocytes in the blood and macrophages in the tissues. Monocytes are produced by bone marrow and remain in the blood for 2-3 days before entering the tissues to further differentiate 
into mature macrophages, which have different types and names in various organs and tissues (7). Recent studies have found that activated macrophages play a significant role in the development of DN and are the major immune cells responsible for renal tissue stromal hyperplasia, glomerular sclerosis and irreversible pathological changes of glomeruli (8-10). Mononuclear macrophages have plasticity and pluripotency, can acquire different phenotypes, and exert distinct immune effects in different environments in the body (11). Specifically, they can be divided into two types in terms of their functions, namely, classical M1 macrophages activated by Th1 cytokines or lipopolysaccharides and Th2 cytokine-derived alternative M2 macrophages (12). M1 and M2 macrophages play opposing roles in the regulation of inflammation. More specifically, M1 macrophages play important roles in antigen presentation and immune inflammatory effects, whereas M2 macrophages mainly release cytokines that inhibit inflammation and exert anti-inflammatory effects $(7,13)$.

As a member of the Sirtuin family, Sirt6 is located on chromosome 19p13.3 (14) and consists of a N-terminal, $\mathrm{C}$-terminal and conserved central domain. The N-terminus is associated with chromosome binding and catalytic activity, while the C-terminus is involved in nuclear localization (15-17). Sirt6 has the activities of $\mathrm{NAD}^{+}$-dependent deacetylase and ADP-ribonyltransferase; therefore, it not only regulates the expression of certain genes, DNA repair and gene stability and the homeostasis of hematopoietic cells, but also maintains glucose homeostasis in the body and plays a role in the occurrence of obesity (17-21). A previous study on the zinc-dependent histone deacetylase revealed that histone deacetylase 4 (HDAC4) can promote the injury of podocytes in DN (22). However, to the best of our knowledge, the roles of class III histone deacetylases, Sirtuins, in the kidneys have not yet been fully investigated. In the present study, we thus aimed to explore the associations among Sirt6, macrophages and DN, and to elucidate the role of Sirt6 in DN.

\section{Materials and methods}

Animal model, tissues samples and monocyte separation. A total of 36 male Sprague-Dawley (SD) rats were purchased from the Animal Experimental Center of Guangdong Medical (Guangdong, China). The rats (weighing 220-250 g) were kept in cages at room temperature $\left(22 \pm 3^{\circ} \mathrm{C}\right)$ with a stable humidity $(50 \pm 10 \%)$ with free access to food/water in a light/dark (12-h/12-h) cycle. The animal experiments were performed according to the Guide for the Care and Use of Laboratory Animals and approved by the First People's Hospital of Yuhang District, Hangzhou Animal Ethics Committee (Hangzhou, China). A diabetic model was established using 18 rats, which were intraperitoneally injected with $60 \mathrm{mg} / \mathrm{kg}$ streptozotocin over a period of 5 consecutive days and streptozotocin was dissolved in citrate buffer, as previously described (23). A total of 18 control rats were intraperitoneal injected with citrate buffer ( $\mathrm{pH} 4.5$ ). At 1, 3 and 5 weeks, blood glucose levels in the rats ( $\mathrm{n}=6$ at each time point) with or without streptozotocin injections were measured by obtaining tail venous blood. Rats with post-fasting blood glucose levels $>250 \mathrm{mg} / \mathrm{dl}$ were defined as diabetic.
The rats $(n=6)$ with or without streptozotocin injections were sacrificed at 1, 3 and 5 weeks and their kidneys were removed and cut into slices. The kidney tissues were divided into two parts; one part was stored in $4 \%$ formaldehyde solution for a routine pathological diagnosis, and the other was stored in $-80^{\circ} \mathrm{C}$ to help perform the following reverse transcription-quantitative PCR (RT-qPCR) and western blot analyses.

Fresh renal tissues that were cut into small sections were transferred to DMEM with collagenase at $37^{\circ} \mathrm{C}$ for $45 \mathrm{~min}$ and filtered through a nylon cell strainer. The digested cells were added to DMEM (Gibco/Thermo Fisher Scientific, Waltham, MA, USA) medium and were centrifuged at 1,200 $\mathrm{x} g$ for $10 \min$ at $4^{\circ} \mathrm{C}(23)$.

Hematoxylin and eosin $(H \& E)$ and immunohistochemical (IHC) staining. Renal tissues specimens were obtained from 4\% paraformaldehyde fixative, and then dehydrated and embedded in paraffin. The embedded tissues were sliced into 4- $\mu$ m-thick sections, which were stained with hematoxylin (Sigma-Aldrich, St. Louis, MO, USA) for $5 \mathrm{~min}$ at room temperature, then stained with eosin (Sigma-Aldrich) for $3 \mathrm{~min}$ at room temperature, and observed under a DMi8 optical microscope (Leica, Hamburg, Germany) at x200 magnification.

Sirt6 expression in the renal tissues from the rats with or without DN were examined by IHC staining. The sections were dewaxed using two types of xylene solution, hydrated by ethanol at various gradients and antigen retrieval was achieved by quenching with endogenous peroxidase. The sections were then washed with PBS for 3 times and incubated with primary Sirt6 antibodies (1:100; ab135566, Abcam, Cambridge, MA, USA) at $4^{\circ} \mathrm{C}$ overnight and then washed with PBS. The sections were then incubated with secondary antibody biotin-conjugated goat anti-rabbit IgG (Proteintech Group, Inc./Thermo Fisher Scientific; SA00004-2; 1:200) at $25^{\circ} \mathrm{C}$ for $30 \mathrm{~min}$. Subsequently, diaminobenzidine (DAB) was used as a chromogen and hematoxylin was used to re-dye the sections, at $25^{\circ} \mathrm{C}$ for $3 \mathrm{~min}$. The stained sections were examined using a DMi8 optical microscope (Leica) in randomly selected sections at x200 magnification.

THP-1 cell culture and macrophage transformation. Human monocyte THP-1 cells were purchased from the Shanghai Cell Bank of Chinese Academy of Sciences (Shanghai, China). The cells were cultured at $37^{\circ} \mathrm{C}$ with $5 \% \mathrm{CO}_{2}$ in RPMI-1640 medium (GENOM, Hangzhou, China) with $10 \%$ fetal bovine serum (Thermo Fisher Scientific), $1 \%$ penicillin/streptomycin (Gibco/Thermo Fisher Scientific). To form macrophages, $100 \mathrm{ng} / \mathrm{ml}$ phorbol 12-myristate 13-acetate (PMA; Sigma-Aldrich), which is also named tetradecanoylphorbol 13-acetate (TPA), was added to the THP-1 cells and cultured at $37^{\circ} \mathrm{C}$ for $48 \mathrm{~h}$. The cells were then washed with PBS twice and continuously cultured in RPMI-1640 medium.

M1 type macrophages were transformed by glucose and incubated at $37^{\circ} \mathrm{C}$ for $24 \mathrm{~h}$. The cells were washed with PBS 3 times and cultured in normal RPMI-1640 meduma for a further $24 \mathrm{~h}$. The supernatants were then collected and centrifuged at $2,000 \times \mathrm{g}$ at $4^{\circ} \mathrm{C}$ for $20 \mathrm{~min}$. There were 4 different groups of cells as follows: The controls (exposed to $5.5 \mathrm{mmol} / 1$ glucose), the HG1 group (exposed to $15 \mathrm{mmol} / \mathrm{l}$ glucose), the HG2 group 
(exposed to $30 \mathrm{mmol} / \mathrm{l}$ glucose) and the HG3 group (exposed to $50 \mathrm{mmol} / 1$ glucose).

Podocyte culture. Immortalized mouse podocyte MPC-5 cells were purchased from the Research Facilities of Peking Union Medical College (PUMC) Cell Bank (Beijing, China). Undifferentiated MPC-5 cells were cultured in a DMEM (Gibco/Thermo Fisher Scientific) with $10 \mathrm{U} / \mathrm{ml}$ mouse recombinant interferon- $\gamma$ (IFN- $\gamma$; Peprotech Inc., Rocky Hill, NJ, USA) at $33^{\circ} \mathrm{C}$ in an incubator with $5 \% \mathrm{CO}_{2}$. Podocytes were cultured at $37^{\circ} \mathrm{C}$ for $10-14$ days in DMEM without IFN- $\gamma$ to induce cell differentiation and maturation.

Cell transfection. Sirt6 overexpression and empty control plasmids were purchased from Invitrogen/Thermo Fisher Scientific. Macrophages and MPC-5 cells were respectively seeded in 6 -well plates $\left(1.0 \times 10^{5}\right)$ for $24 \mathrm{~h}$ prior to transfection. Transient transfection was carried out using Lipofectamine 3000 (Invitrogen/Thermo Fisher Scientific) according to the standard protocol. A total of $20 \mu \mathrm{M}$ overexpressing-RNA, control, negative control (NC) and Lipofectamine 3000 were added to serum-free medium and incubated at $25^{\circ} \mathrm{C}$ for $10 \mathrm{~min}$, respectively. Subsequently, Lipofectamine 3000 was mixed into each group that was cultured in serum-free DMEM. Following $6 \mathrm{~h}$ of culture, the fluid was replaced by DMEM containing $10 \%$ fetal bovine serum (FBS).

Co-culture of macrophages with podocytes. We used a Transwell to establish a co-culture system. Macrophages were transfected with control/NC/Sirt6 overexpression plasmid and seeded on a $0.4 \mu \mathrm{m}$ Transwell insert (Millipore, Billerica, MA, USA) with RPMI-1640 medium and then co-cultured with podocytes transfected with control/NC/Sirt6 overexpression plasmids for $48 \mathrm{~h}$ in the presence of $50 \mathrm{mmol} / \mathrm{l}$ high-glucose treatment. In this experiment, the cells were divided into 8 groups as follows: i) The $\mathrm{NM}+\mathrm{HG}+$ Con group (control; $50 \mathrm{mmol} / 1$ of high-glucose medium in the upper chamber, and podocytes with high-glucose medium in the lower chamber); ii) the $\mathrm{HG}+$ Con group (macrophages with high-glucose medium in the upper chamber, and podocyte with high-glucose medium in the lower chamber); iii) the $\mathrm{HG}+\mathrm{NC}+$ Con group (macrophages transfected with $\mathrm{NC}$ plasmid with high-glucose medium in the upper chamber, and podocytes with high-glucose medium in the lower chamber); iv) the HG + Sirt6 + Con group (macrophages transfected with Sirt6 overexpression plasmid with high-glucose medium in the upper chamber, and podocytes with high-glucose medium in the lower chamber); v) the M-Sirt6 group (high-glucose medium in the upper chamber, and podocytes transfected with Sirt6 overexpression plasmid with high-glucose medium in the lower chamber); vi) the HG + M-Sirt6 group (macrophages with high-glucose medium in the upper chamber, and podocytes transfected with Sirt6 overexpression plasmid with high-glucose medium in the lower chamber); vii) the $\mathrm{HG}+\mathrm{NC}+\mathrm{M}-\mathrm{Sirt6}$ group (macrophages transfected with $\mathrm{NC}$ plasmid with high-glucose medium in the upper chamber, and podocytes transfected with Sirt6 overexpression plasmid with high-glucose medium in the lower chamber); and viii) the HG + Sirt6 + M-Sirt6 (macrophages transfected with Sirt6 overexpression plasmid with high-glucose medium in the upper chamber, and podocytes transfected with Sirt6 overexpression plasmid with high-glucose medium in the lower chamber). The control group and M-Sirt6 (macrophages with high-glucose medium in the upper chamber, and podocytes transfected with Sirt6 overexpression plasmid with high-glucose medium in the lower chamber) group were not cultured with macrophages.

Flow cytometry. Cell apoptosis was determined by flow cytometry with the Annexin V-FITC/PI staining kit (BD Biosciences, San Jose, CA, USA). The cells were washed twice using washing buffer, and the suspension was cultured with Annexin V and propidium iodide (PI; Yeasen Biotechnology Co., Ltd., Shanghai, China) in the dark at $25^{\circ} \mathrm{C}$ for $15 \mathrm{~min}$. Binding buffer was then added into each well. The samples were analyzed by BD FACSCalibur flow cytometer (BD Biosciences, San Diego, CA, USA) within $1 \mathrm{~h}$.

$R T$ - $q P C R$. Sirt6, tumor necrosis factor (TNF)- $\alpha$, inducible nitric oxide synthase (iNOS), interleukin (IL)-4, IL-10, CD86, CD206, Bcl-2 and Bax expression levels were detected by RT-qPCR in the different groups. Total RNA was obtained from the tissues or cultured cells using TRIzol reagent (Invitrogen/Thermo Fisher Scientific) according to the manufacturer's instructions. Reverse transcription was performed using the PrimeScript ${ }^{\mathrm{TM}}$ RT reagent kit (Takara, Shiga, Japan) at $37^{\circ} \mathrm{C}$ for $15 \mathrm{~min}$ and $85^{\circ} \mathrm{C}$ for $5 \mathrm{~min}$. cDNA amplified using SYBR Fast qPCR Mix (Invitrogen/Thermo Fisher Scientific) and the Sirt6 cycle was set at $95^{\circ} \mathrm{C}$ for $5 \mathrm{~min}$, followed by 30 cycles at $95^{\circ} \mathrm{C}$ for $30 \mathrm{sec}$, at $58^{\circ} \mathrm{C}$ for $30 \mathrm{sec}$ and at $72^{\circ} \mathrm{C}$ for $30 \mathrm{sec}$. The TNF- $\alpha$ cycle was set at $94^{\circ} \mathrm{C}$ for $5 \mathrm{~min}$, followed by 40 cycles at $94^{\circ} \mathrm{C}$ for $30 \mathrm{sec}$, at $60^{\circ} \mathrm{C}$ for $30 \mathrm{sec}$ and at $72^{\circ} \mathrm{C}$ for $60 \mathrm{sec}$. The iNOS cycle was set at $94^{\circ} \mathrm{C}$ for $5 \mathrm{~min}$, followed by 35 cycles at $94^{\circ} \mathrm{C}$ for $30 \mathrm{sec}$, at $62^{\circ} \mathrm{C}$ for $30 \mathrm{sec}$ and at $72^{\circ} \mathrm{C}$ for $30 \mathrm{sec}$. The IL- 4 cycle was set at $94^{\circ} \mathrm{C}$ for $4 \mathrm{~min}$, followed by 35 cycles at $94^{\circ} \mathrm{C}$ for $30 \mathrm{sec}$, at $54^{\circ} \mathrm{C}$ for $30 \mathrm{sec}$ and at $72^{\circ} \mathrm{C}$ for $40 \mathrm{sec}$. The IL-10 cycle was set at $95^{\circ} \mathrm{C}$ for $3 \mathrm{~min}$, followed by 45 cycles at $95^{\circ} \mathrm{C}$ for $15 \mathrm{sec}$, at $53^{\circ} \mathrm{C}$ for $30 \mathrm{sec}$ and at $60^{\circ} \mathrm{C}$ for $30 \mathrm{sec}$. The Bax, Bcl-2 and GAPDH cycles were set at $95^{\circ} \mathrm{C}$ for $10 \mathrm{sec}$, followed by 40 cycles at $95^{\circ} \mathrm{C}$ for $30 \mathrm{sec}$, at $58^{\circ} \mathrm{C}$ for $15 \mathrm{sec}$ and at $72^{\circ} \mathrm{C}$ for $15 \mathrm{sec}$. GAPDH served as an internal control. All primers were used in the synthesis (Sangon Biotech, Shanghai, China) and sequences are listed in Table I. Amplified products were electrophoresed through $2 \%$ agarose gels. The amount of RNA was quantified using the $2^{-\Delta \Delta \mathrm{Ct}}$ method (24).

Western blot analysis. Sirt6, Nephrin, Desmin, CD86, CD206 proteins were extracted from the tissues or cells using RIPA lysis buffer (Beyotime, Shanghai, China). The concentrations of proteins were detected using the BCA protein kit (Beyotime, Shanghai, China). Aliquot protein was separated by $12 \%$ SDS-PAGE and resolved proteins were transferred onto polyvinylidene fluoride (PVDF) membranes (Millipore), which were blocked in 5\% skim milk PBS with $0.1 \%$ Triton X-100 and incubated with primary antibodies as follows: anti-Nephrin antibody (1:1,000; ab216341), anti-Desmin antibody (1:1,000; ab15200), anti-Sirt6 antibody (1:2,000; ab191385), anti-CD86 antibody (1:5,000; ab53004 and ab112490), anti-CD206 antibody (1:1,000; ab64693), anti-Bcl-2 antibody (1:2,000; ab182858) and anti-Bax antibody (1:1,000; ab32503) (all 
Table I. Primers used in RT-qPCR.

\begin{tabular}{|c|c|c|c|}
\hline Gene & Species & Primer & Sequence \\
\hline TNF- $\alpha$ & Rat & $\begin{array}{l}\text { Forward } \\
\text { Reverse }\end{array}$ & $\begin{array}{l}\text { 5'-CCAACAAGGAGGAGAAGTTCC-3' } \\
\text { 5'-TCTGCTTGGTGGTTTGCTAC-3' }\end{array}$ \\
\hline iNOS & Rat & $\begin{array}{l}\text { Forward } \\
\text { Reverse }\end{array}$ & $\begin{array}{l}\text { 5'-GGTGTTCTTTGCTTCTGTGCTA-3' } \\
\text { 5'-CTTCCGACTTTCCTGTCTCAGT-3' }\end{array}$ \\
\hline iNOS & Human & $\begin{array}{l}\text { Forward } \\
\text { Reverse }\end{array}$ & $\begin{array}{l}\text { 5'-CCAGCTAGCCAAAGTCACCAT-3' } \\
\text { 5'-GTCTCGGAGCCATACAGGATT-3' }\end{array}$ \\
\hline IL-4 & Rat & $\begin{array}{l}\text { Forward } \\
\text { Reverse }\end{array}$ & $\begin{array}{l}\text { 5'-TTACCCGTCTCATTTGC-3' } \\
\text { 5'-TTCCCACTTTGCTGTTCC-3' }\end{array}$ \\
\hline IL-10 & Rat & $\begin{array}{l}\text { Forward } \\
\text { Reverse }\end{array}$ & $\begin{array}{l}\text { 5'-CAGAAATCAAGGAGCATTTG-3' } \\
\text { 5'-CTGCTCCACTGCCTTGCTTT-3' }\end{array}$ \\
\hline IL-10 & Human & $\begin{array}{l}\text { Forward } \\
\text { Reverse }\end{array}$ & $\begin{array}{l}\text { 5'-GCCAAGCCTTGTCTGAGATGATCC-3' } \\
\text { 5'-AATCGATGACAGCGCCGTAGC-3' }\end{array}$ \\
\hline Sirt6 & Human & $\begin{array}{l}\text { Forward } \\
\text { Reverse }\end{array}$ & $\begin{array}{l}\text { 5'- AATTACGCGGCGGGGCT-3' } \\
\text { 5'-CGCGCGCTCTCAAAGGT-3' }\end{array}$ \\
\hline Sirt6 & Mouse & $\begin{array}{l}\text { Forward } \\
\text { Reverse }\end{array}$ & $\begin{array}{l}\text { 5'-GGCTACGTGGATGAGGTGAT-3' } \\
\text { 5'-GGCTCAGCCTTGAGTGCTAC-3' }\end{array}$ \\
\hline Bax & Mouse & $\begin{array}{l}\text { Forward } \\
\text { Reverse }\end{array}$ & $\begin{array}{l}\text { 5'-AGCCACAAAGATGGTCACT-3' } \\
\text { 5'-GGAGATGAACTGGATAGCAA-3' }\end{array}$ \\
\hline Bcl-2 & Mouse & $\begin{array}{l}\text { Forward } \\
\text { Reverse }\end{array}$ & $\begin{array}{l}\text { 5'-ATCTCCCTGTTGACGCTCT-3' } \\
\text { 5'-CATCTTCTCCTTCCAGCCT-3' }\end{array}$ \\
\hline GAPDH & Human & $\begin{array}{l}\text { Forward } \\
\text { Reverse }\end{array}$ & $\begin{array}{l}\text { 5'-ACCACAGTCCATGCCATCAC-3' } \\
\text { 5'-TCCACCACCCTGTTGCTGTA-3' }\end{array}$ \\
\hline
\end{tabular}

from Abcam) overnight at $4^{\circ} \mathrm{C}$. The membranes were then incubated with the appropriate HRP-conjugated secondary antibody (Proteintech Group, Inc./Thermo Fisher Scientific, SA00001-2, 1:5,000). Protein bands were detected with ECL (Thermo Fisher Scientific) and visualized using Quantity One software (Bio-Rad, Hercules, CA, USA).

Statistical analysis. Statistical analysis was carried out Prism GraphPad version 6.0 software. All data were presented as the means \pm standard deviation (SD). Differences were analyzed using one-way analysis of variance (ANOVA) followed by Tukey's multiple comparisons test. A value of $\mathrm{P}<0.05$ was considered to indicate a statistically significant difference.

\section{Results}

Podocyte injury and low expression of Sirt6 in DN rat model. The fasting blood glucose levels of all the rats administered streptozotocin were $>250 \mathrm{mg} / \mathrm{dl}$, indicating that the rat model of diabetes was successfully established. Moreover, the glucose levels of the rats administered streptozotocin were noticeably higher than those of the rats not administered streptozotocin $(\mathrm{P}<0.01$; Fig. $1 \mathrm{~A})$. H\&E staining revealed that in the DN group, the glomerular local basement membranes had become thickened, and the phenomena of mesangial matrix hyperplasia and renal interstitial fibrosis were observed as well, compared to the controls (Fig. 1B). The expression of Sirt6 in the renal tissues was examined by IHC staining. As shown in Fig. 1C, Sirt6 protein expression was positive in the control group; however, the expression of Sirt6 was downregulated in the rats with DN (Fig. 1C). Western blot analysis was also used to detect the protein expression levels of Nephrin, the podocyte injury marker, Desmin, and Sirt6. The results revealed that the protein levels of Desmin markedly increased, and the protein expression levels of both Nephrin and Sirt6 significantly decreased in the tissues of the rats with $\mathrm{DN}(\mathrm{P}<0.01$; Fig. 1D), indicating that streptozotocin-induced DN can lead to podocyte injury and a low expression of Sirt6.

Immune function of macrophages in rats with streptozotocin-induced DN. Monocytes were separated from the rat kidneys. Different types of monocytes and Sirt6 expression were then examined by western blot analysis. Compared to the controls, the expression of the marker of the M1 type of monocytes, CD86, was significantly upregulated, whereas that of the marker of the M2 type of monocytes, CD206, was significantly downregulated in the rats with $\mathrm{DN}(\mathrm{P}<0.01$; Fig. 1E). In addition, the protein expression of Sirt6 was notably decreased in the DN group $(\mathrm{P}<0.01$; Fig. 1E). Moreover, the levels of inflammatory factors were determined to assess the immune function of macrophages. The results revealed that the mRNA expression levels of the pro-inflammatory cytokines, TNF- $\alpha$ and iNOS, were significantly increased in the rats with DN compared with the controls $(\mathrm{P}<0.01$; Fig. 1F). However, the mRNA expression levels of IL-4 and IL-10 were significantly 
A

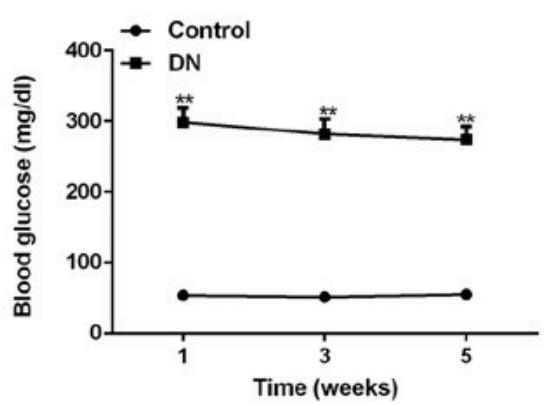

B

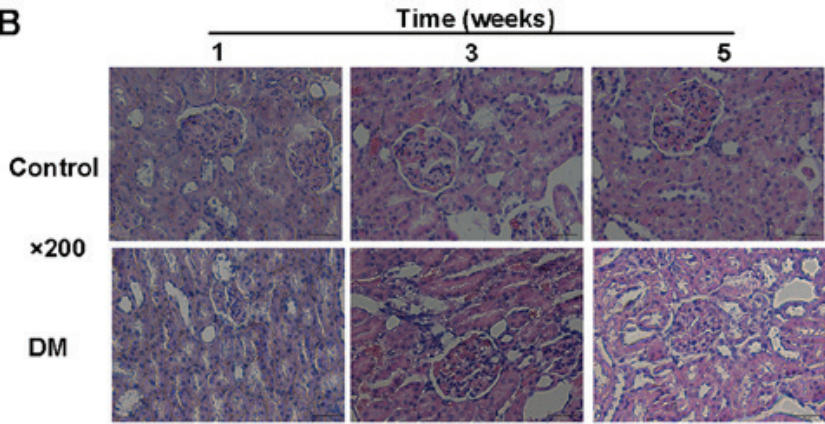

C

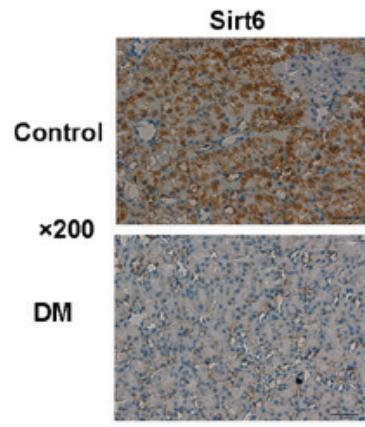

E

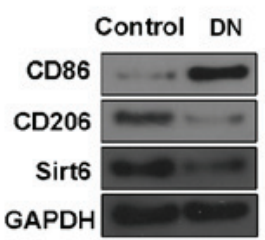

D
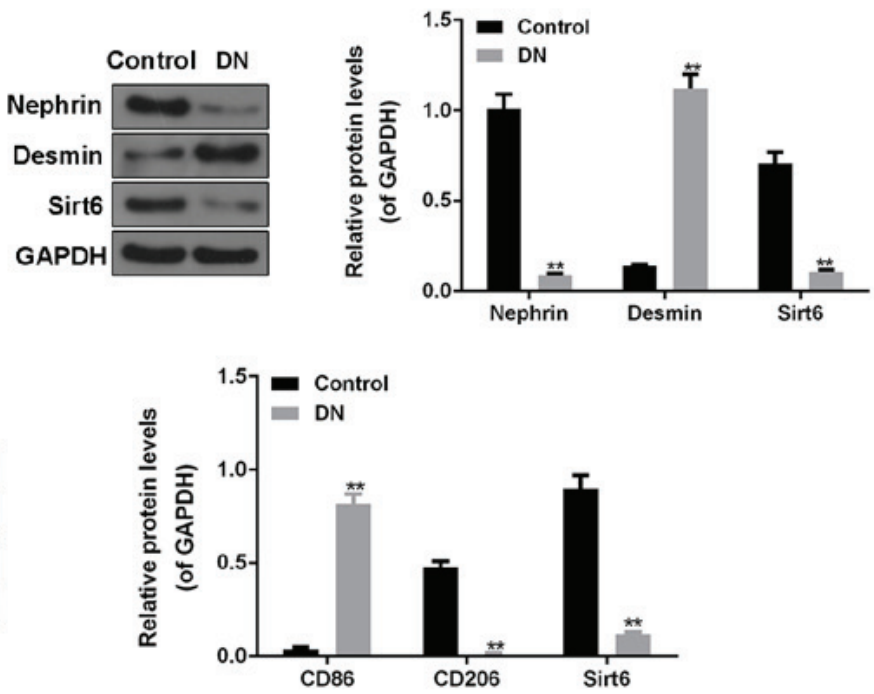
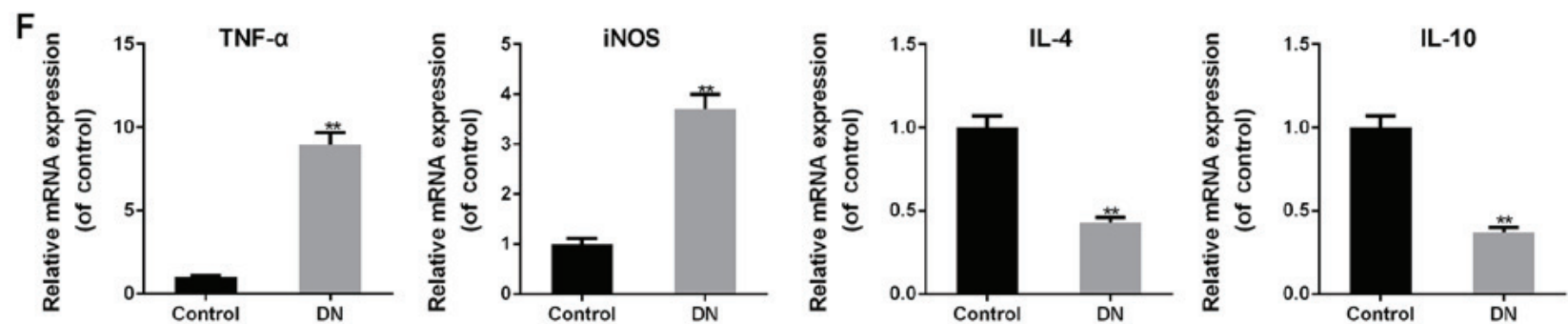

Figure 1. Streptozotocin-induced diabetic nephropathy (DN) in rats leads to podocyte injury, a low expression of Sirt6 and the immune function of macrophages. (A) Fasting plasma glucose levels in rats with or without streptozotocin treatment were measured by a glucometer at 1, 3 and 5 weeks. Post-fasting blood glucose levels $>250 \mathrm{mg} / \mathrm{dl}$ were defined as diabetic. (B) Hematoxylin and eosin (H\&E) staining was performed to observe the renal tissues of normal rats and rats with DN rats at 1,3 and 5 weeks by an optical microscope at x200 magnification. (C) Immunohistochemical (IHC) staining was performed to obverse the protein expression of Sirt6 by an optical microscope at x200 magnification. (D) Western blot analysis was performed to assess the protein expression levels of Nephrin, Desmin and Sirt6 in the control rats and rats with DN. (E) Western blot analysis was performed to assess the protein expression levels of CD86, CD206 and Sirt6 in the control rats and rats with DN. GAPDH was used as an internal control. (F) RT-qPCR was used to detect the mRNA expression levels of TNF- $\alpha$, iNOS, IL-4 and IL-10 in the control rats and rats with DN. Data are presented as the means \pm SD from 3 independent experiments ("* P $<0.01$, compared with the controls).

decreased in the rats with $\mathrm{DN}$ in comparison with the controls $(\mathrm{P}<0.01$; Fig. 1F).

Macrophages undergo M1 transformation under high-glucose conditions in a dose-dependent manner. In order to examine the effects of glucose treatment on macrophage phenotypes, the typical markers of M1 and M2 macrophages, and related inflammatory factors were analyzed. The results revealed that the macrophages exhibited increased mRNA expression levels of CD86 (HG1, P<0.05; HG2 and HG3, P<0.01; Fig. 2A) and
iNOS ( $<<0.01$; Fig. $2 \mathrm{C}$ ) as the glucose concentrations increased. However, the mRNA level of CD206 did not differ significantly between the controls and the $3 \mathrm{HG}$ groups (macrophages were exposed to 3 different concentrations of glucose) $(\mathrm{P}>0.05$; Fig. 2B). The mRNA levels of IL-10 (HG1, P<0.05; HG2 and HG3, P<0.01; Fig. 2D) and Sirt6 (HG2 and HG3, P<0.01; Fig. 2E) significantly decreased by exposing the cells to glucose in a dose-dependent manner. These results illustrate that high glucose induces the transformation of macrophages into the M1 phenotype and decreases the expression of Sirt6 in macrophages. 

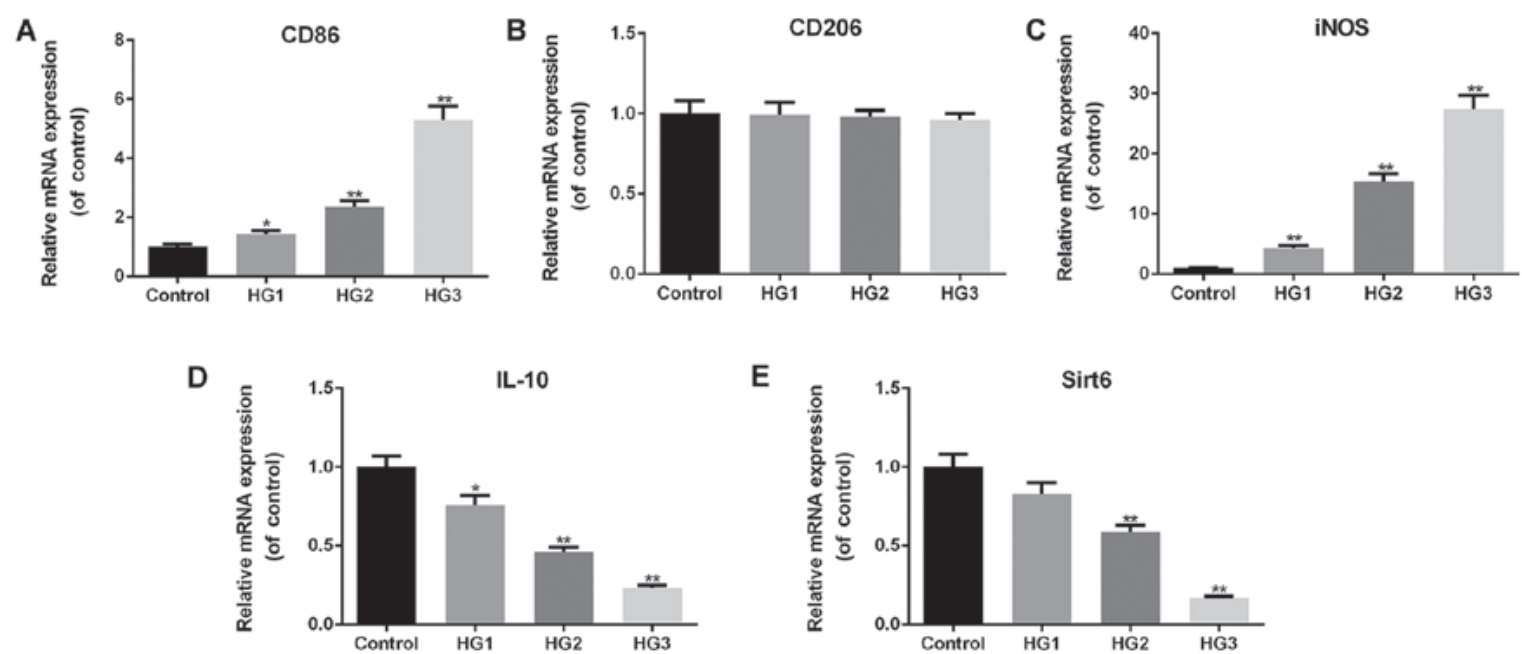

Figure 2. Macrophages undergo M1 transformation under high-glucose conditions. RT-qPCR was performed to examine the effects of various concentrations of glucose on the mRNA expression levels of (A) CD86, (B) CD206, (C) iNOS, (D) IL-10, and (E) Sirt6. The cell groups were as follows: The control, $5.5 \mathrm{mmol} / \mathrm{l}$ glucose; HG1, $15 \mathrm{mmol} / \mathrm{l}$ glucose; HG2, $30 \mathrm{mmol} / 1$ glucose; HG3, $50 \mathrm{mmol} / \mathrm{l}$ glucose. Data are presented as the means $\pm \mathrm{SD}$ from 3 independent experiments ( $\mathrm{P}<0.05$ and ${ }^{* *} \mathrm{P}<0.01$, compared with the controls).

A
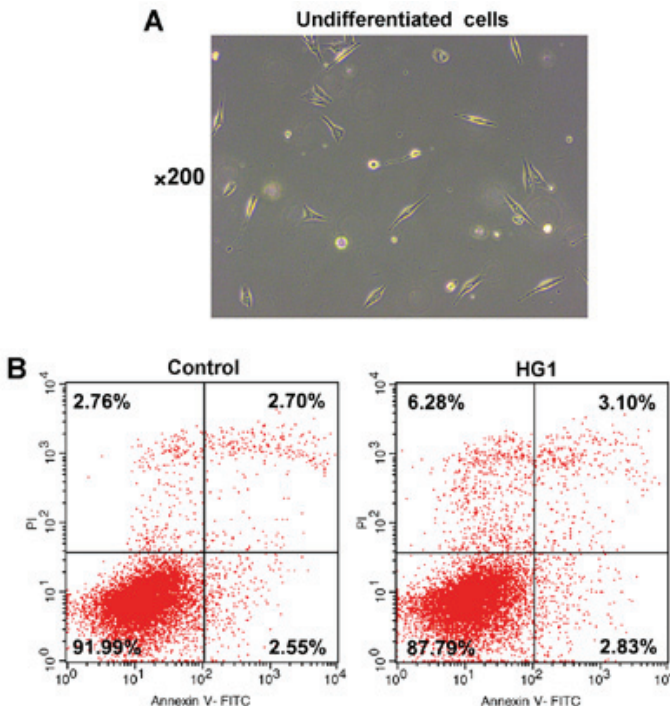

C

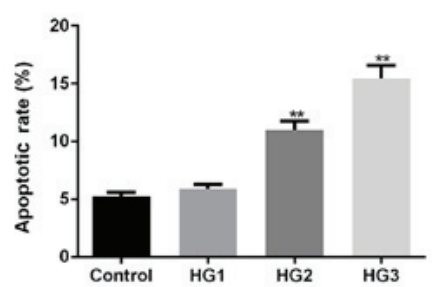

E

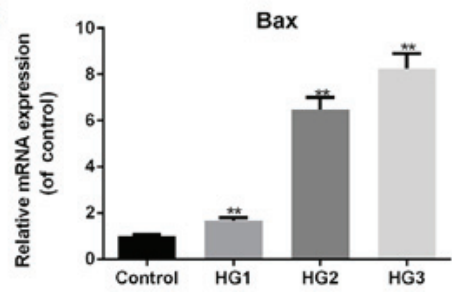

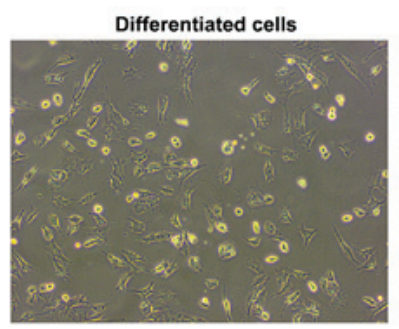
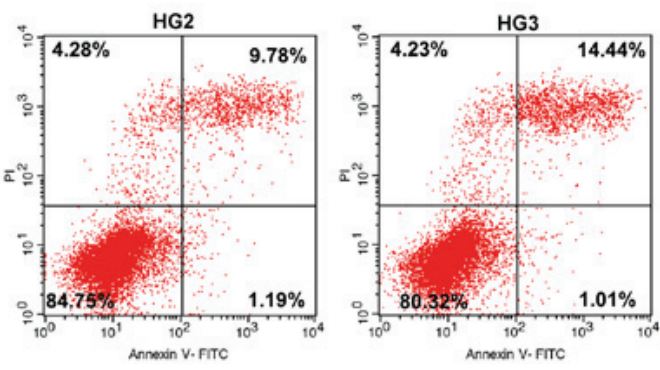

D

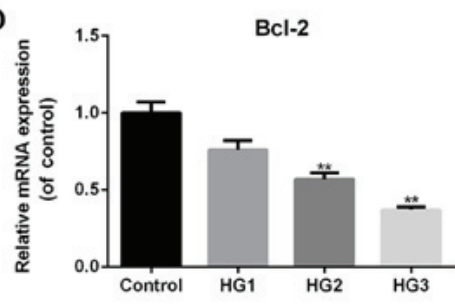

$\mathbf{F}$

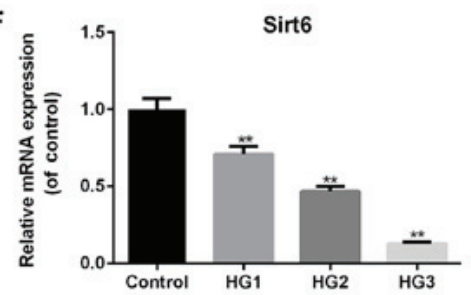

Figure 3. Glucose promotes podocyte apoptosis in a dose-dependent manner. (A) Morphological observation of MPC-5 podocytes under an inverted microscope (x200 magnification) under the undifferentiated and differentiated conditions. (B) Flow cytometry was performed to examine the effects of various concentrations of glucose on the apoptosis of differentiated podocytes. (C) The apoptotic rate is presented as bar diagrams. RT-qPCR was performed to examine the effects of various concentrations of glucose on the mRNA expression levels of (D) Bcl-2, (E) Bax and (F) Sirt6. The cell groups were as follows: The control, $5.5 \mathrm{mmol} / 1$ glucose; HG1, $15 \mathrm{mmol} / 1$ glucose; HG2, $30 \mathrm{mmol} / 1$ glucose; HG3, $50 \mathrm{mmol} / \mathrm{l}$ glucose. Data are presented as the means $\pm \mathrm{SD}$ from 3 independent experiments ( ${ }^{* *} \mathrm{P}<0.01$, compared with the controls). 

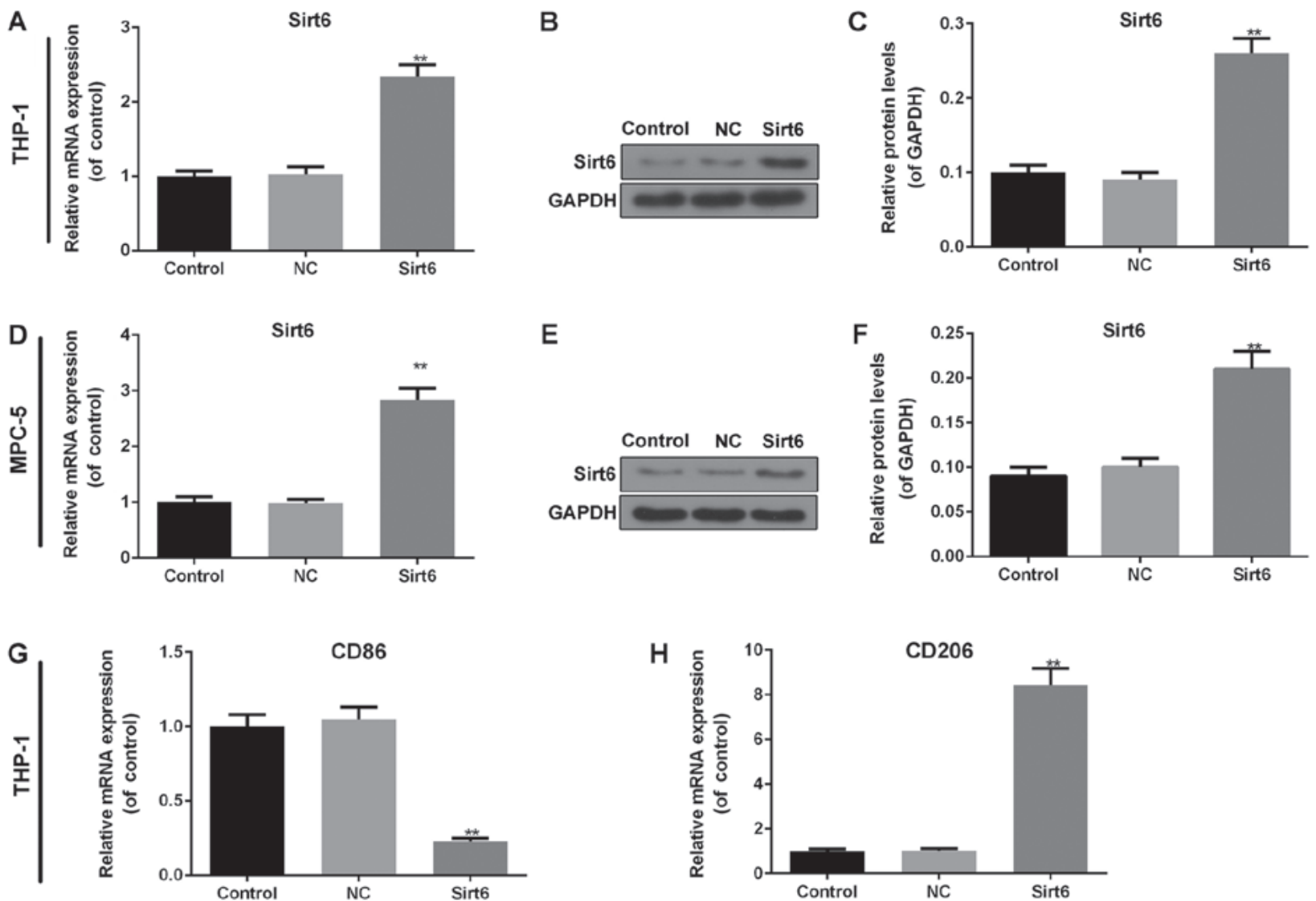

Figure 4. Overexpression of Sirt6 induces macrophage M2 transformation. In macrophages, (A) RT-qPCR and (B and C) western blot analysis were carried out to detect the transfection efficiency following the overexpression of Sirt6. In podocytes, (D) RT-qPCR and (E and F) western blot analysis were used to determine the transfection efficiency of the overexpression of Sirt6. GAPDH was used as an internal control. RT-qPCR was performed to examine the effects of the overexpression of Sirt6 on the mRNA expression levels of $(\mathrm{G}) \mathrm{CD} 86$ and $(\mathrm{H}) \mathrm{CD} 206$ in macrophages. Data are presented as the means \pm SD from 3 independent experiments ( ${ }^{* *} \mathrm{P}<0.01$, compared with the controls).

High glucose promotes podocyte apoptosis in adose-dependent manner. As shown in Fig. 3A, the undifferentiated podocytes exhibited a fusiform or triangular shape. Following subculture, the cells ceased to proliferate, grew larger in size, and gradually differentiated and matured (Fig. 3A). Flow cytometry was used to examine the effects of various concentrations of glucose on the apoptosis of differentiated podocytes (Fig. 3B and C). As the glucose concentrations increased, podocyte apoptosis gradually increased in a dose-dependent manner (HG2 and HG3, P<0.01; Fig. 3C). We also examined the expression of apoptosis-related genes and Sirt6, and the results revealed that the mRNA expression of pro-apoptotic Bax $(\mathrm{P}<0.01$; Fig. $3 \mathrm{E})$ significantly increased, whereas that of Bcl-2 (HG2 and HG3, P<0.01; Fig. 3D) and Sirt6 ( $<<0.01$, Fig. 3F) markedly decreased by exposure to high-glucose concentrations.

Overexpression of Sirt6 induces macrophage M2 transformation. The overexpression of Sirt6 was successfully induced in the macrophages and podocytes by transfection with a Sirt6 overexpression plasmid. In the macrophages, both the mRNA (Fig. 4A) and protein (Fig. 4B and C) levels of Sirt6 exhibited a noticeable upregulation in comparison with the controls or the $\mathrm{NC}$ group $(\mathrm{P}<0.01)$. In addition, in the podocytes, the expression of Sirt6 was consistent with that observed in the macrophages $(\mathrm{P}<0.01$; Fig. $4 \mathrm{D}-\mathrm{F})$. Considering the effects of the overexpression of Sirt6 on macrophage phenotypes,
RT-qPCR was performed to detect the mRNA levels of markers in macrophages. The results revealed that the overexpression of Sirt6 significantly decreased the mRNA level of CD86 $(\mathrm{P}<0.01$; Fig. 4G) and increased that of CD206 (P<0.01; Fig. 4H) compared to the controls, indicating that the overexpression of Sirt6 induced macrophage M2 transformation.

Overexpression of Sirt6 in macrophages inhibits podocyte apoptosis under high-glucose conditions. In order to examine the effects of the overexpression of Sirt6 on podocyte apoptosis, we mimicked a diabetic internal environment using a Transwell co-culture system under high-glucose conditions to co-culture the macrophages and podocytes. Moreover, flow cytometry was performed to detect the apoptotic level (Fig. 5A). Compared to the $\mathrm{NM}+\mathrm{HG}+$ Con group [high-glucose medium (50 $\mathrm{mmol} / \mathrm{l})$ in the upper chamber and podocytes with high-glucose medium in lower chamber], apoptosis was significantly increased in the $\mathrm{HG}+$ Con group (macrophages with high-glucose medium in upper chamber, podocytes with high-glucose medium in lower chamber), indicating that the macrophages significantly enhanced podocyte apoptosis in a high-glucose environment $(\mathrm{P}<0.01$; Fig. 5B). When the overexpression of Sirt6 was induced in the macrophages by transfection with a Sirt6 overexpression plasmid, podocyte apoptosis markedly decreased, compared to that observed in the group with macrophages transfected with the NC plasmid $(\mathrm{P}<0.01)$. However, once Sirt6 was 

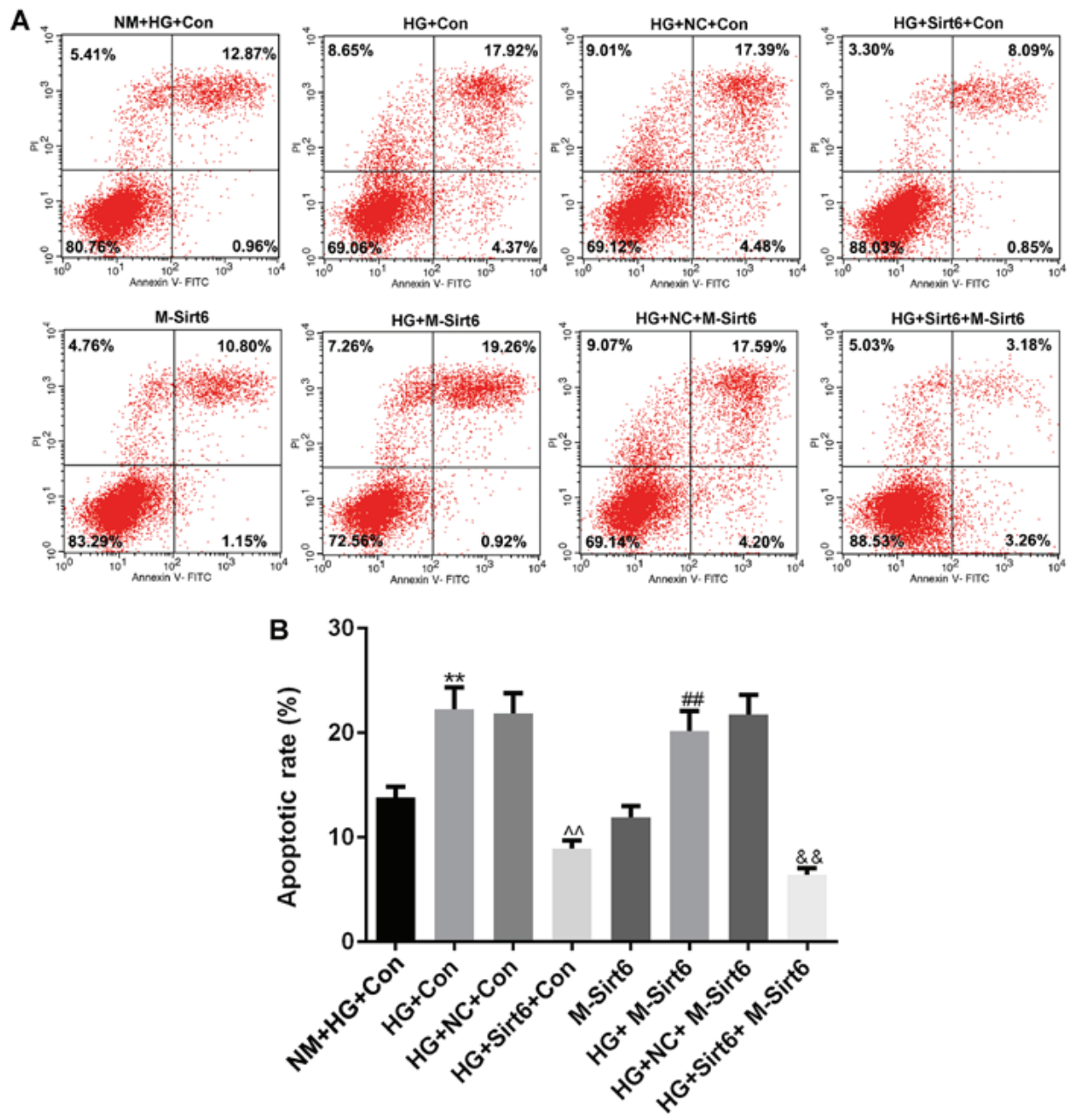

Figure 5. Overexpression of Sirt6 in macrophages inhibits podocyte apoptosis under high-glucose conditions. The diabetic internal environment was mimicked by a Transwell co-culture system under high-glucose conditions to co-culture the macrophages and podocytes. The cells in this experiment were divided into 8 groups as follows: i) The $\mathrm{NM}+\mathrm{HG}+$ Con group (control; $50 \mathrm{mmol} / \mathrm{l}$ of high-glucose medium in the upper chamber, and podocytes with high-glucose medium in the lower chamber); ii) the HG + Con group (macrophages with high-glucose medium in the upper chamber, and podocyte with high-glucose medium in the lower chamber); iii) the $\mathrm{HG}+\mathrm{NC}+\mathrm{Con}$ group (macrophages transfected with $\mathrm{NC}$ plasmid with high-glucose medium in the upper chamber, and podocytes with high-glucose medium in the lower chamber); iv) the HG + Sirt6 + Con group (macrophages transfected with Sirt6 overexpression plasmid with high-glucose medium in the upper chamber, and podocytes with high-glucose medium in the lower chamber); v) the M-Sirt6 group (high-glucose medium in the upper chamber, and podocytes transfected with Sirt6 overexpression plasmid with high-glucose medium in the lower chamber); vi) the HG + M-Sirt6 group (macrophages with high-glucose medium in the upper chamber, and podocytes transfected with Sirt6 overexpression plasmid with high-glucose medium in the lower chamber); vii) the $\mathrm{HG}+\mathrm{NC}+\mathrm{M}$-Sirt6 group (macrophages transfected with $\mathrm{NC}$ plasmid with high-glucose medium in the upper chamber, and podocytes transfected with Sirt6 overexpression plasmid with high-glucose medium in the lower chamber); and viii) the HG + Sirt6 + M-Sirt6 (macrophages transfected with Sirt6 overexpression plasmid with high-glucose medium in the upper chamber, and podocytes transfected with Sirt6 overexpression plasmid with high-glucose medium in the lower chamber). (A) Flow cytometry was performed to examine the effect of the overexpression of Sirt6 on podocyte apoptosis in the Transwell co-culture system. (B) Apoptotic rates are presented as bar diagrams. Data are presented as the means \pm SD from 3 independent experiments $\left({ }^{* *} \mathrm{P}<0.01\right.$, compared with the $\mathrm{NM}+\mathrm{HG}+$ Con group; ${ }^{\wedge} \mathrm{P}<0.01$, compared with the $\mathrm{HG}+\mathrm{NC}+$ Con group; ${ }^{\# /} \mathrm{P}<0.01$, compared with the $\mathrm{M}-\mathrm{Sirt} 6$ group; ${ }^{\&} \mathrm{P}<0.01$, compared with the HG $+\mathrm{M}-$ Sirt6 group).

overexpressed in the podocytes, no significant differences were observed in podocyte apoptosis between the M-Sirt6 group and the $\mathrm{NM}+\mathrm{HG}+$ Con group $(\mathrm{P}>0.05)$. Of note, the apoptosis of the podocytes overexpressing Sirt6 (induced by transfection with a Sirt6 overexpression plasmid) still increased when these podocytes were co-cultured with macrophages in high-glucose medium (HG + M-Sirt6 group) $(\mathrm{P}<0.01)$. However, cell apoptosis was significantly decreased when the podocytes overexpressing Sirt6 were co-cultured with macrophages also overexpressing Sirt6 by transfection with a Sirt6 overexpression plasmid (HG+Sirt6+M-Sirt6 group) $(\mathrm{P}<0.01)$. The above-mentioned results suggested that the overexpression of Sirt6 indirectly affected podocyte apoptosis.
Furthermore, we detected the expression levels of apoptosis-related genes and Sirt6 in podocytes. Under high-glucose conditions, once the macrophages were transfected with a Sirt6 overexpression plasmid, the mRNA expression of $\mathrm{Bcl}-2$ increased $(\mathrm{P}<0.01$; Fig. $6 \mathrm{~A})$ and that of $\mathrm{Bax}$ expression decreased $(\mathrm{P}<0.01$; Fig. $6 \mathrm{~B})$. As regards the mRNA expression of Sirt6, the results revealed that the Sirt6 mRNA level was upregulated when Sirt6 was overexpressed in the podocytes (Fig. 6C). The protein levels of Bcl-2, Bax and Sirt6 were similar to the mRNA levels (Fig. 6D and E).

Moreover, the macrophage phenotypes and the expression of Sirt6 in the macrophages were subsequently detected in the mimicked diabetic internal environment in order to examine 

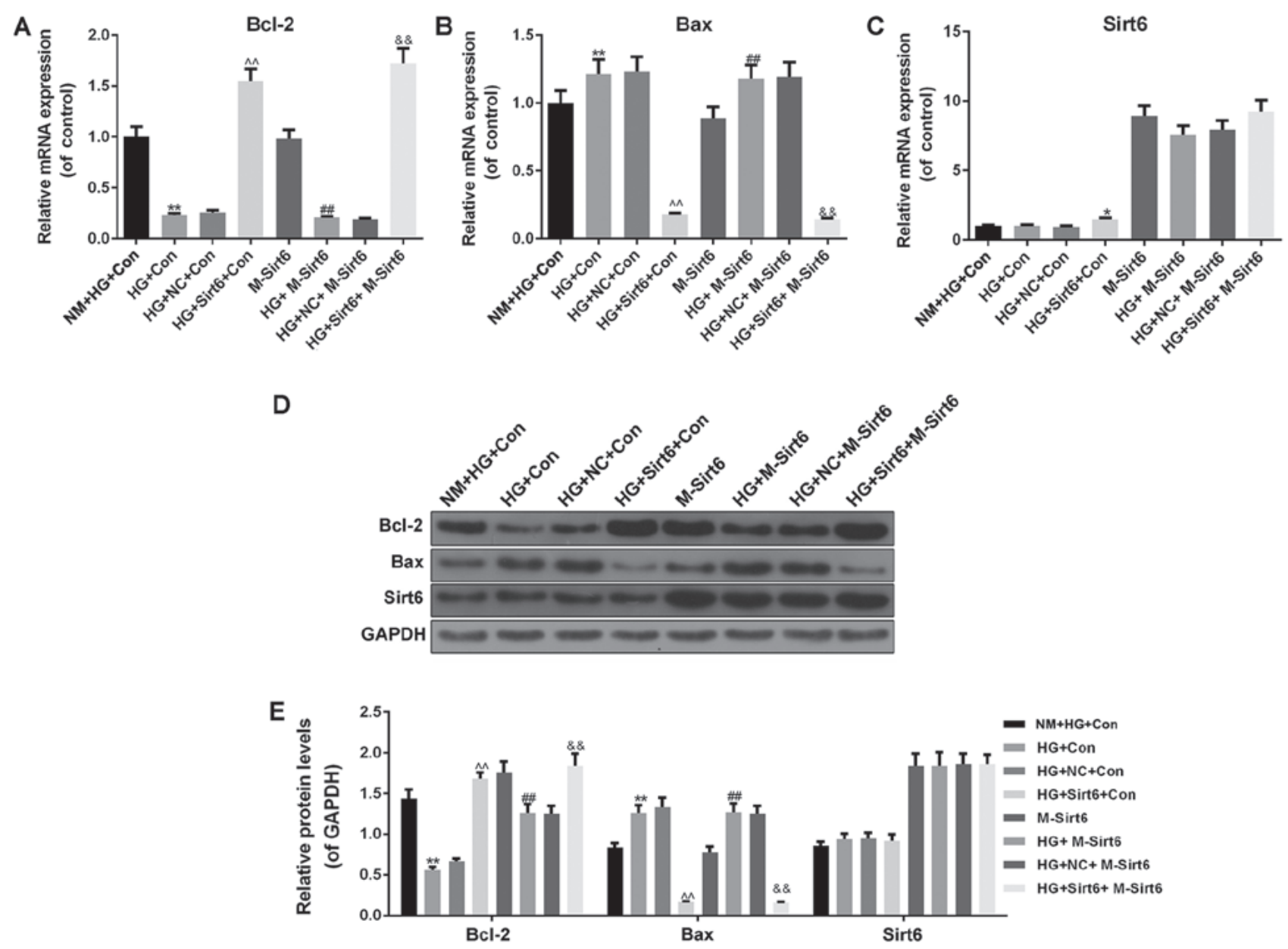

Figure 6. Overexpression of Sirt6 in macrophages regulates the expression of apoptosis-related genes and Sirt6 in podocytes under high-glucose conditions. The diabetic internal environment was mimicked by a Transwell co-culture system under high-glucose conditions to co-culture the macrophages and podocytes. The cells in this experiment were divided into 8 groups as follows: i) The NM + HG + Con group (control; 50 mmol/1 of high-glucose medium in the upper chamber, and podocytes with high-glucose medium in the lower chamber); ii) the HG + Con group (macrophages with high-glucose medium in the upper chamber, and podocyte with high-glucose medium in the lower chamber); iii) the $\mathrm{HG}+\mathrm{NC}+$ Con group (macrophages transfected with $\mathrm{NC}$ plasmid with high-glucose medium in the upper chamber, and podocytes with high-glucose medium in the lower chamber); iv) the HG + Sirt6 + Con group (macrophages transfected with Sirt6 overexpression plasmid with high-glucose medium in the upper chamber, and podocytes with high-glucose medium in the lower chamber); v) the M-Sirt6 group (high-glucose medium in the upper chamber, and podocytes transfected with Sirt6 overexpression plasmid with high-glucose medium in the lower chamber); vi) the HG + M-Sirt6 group (macrophages with high-glucose medium in the upper chamber, and podocytes transfected with Sirt6 overexpression plasmid with high-glucose medium in the lower chamber); vii) the HG + NC + M-Sirt6 group (macrophages transfected with NC plasmid with high-glucose medium in the upper chamber, and podocytes transfected with Sirt6 overexpression plasmid with high-glucose medium in the lower chamber); and viii) the HG + Sirt6 + M-Sirt6 (macrophages transfected with Sirt6 overexpression plasmid with high-glucose medium in the upper chamber, and podocytes transfected with Sirt6 overexpression plasmid with high-glucose medium in the lower chamber). RT-qPCR was performed to examine the effects of the overexpression of Sirt6 on the mRNA expression levels of (A) Bcl-2, (B) Bax and (C) Sirt6 in the Transwell co-culture system. (D) Western blot analysis was performed to examine the effects of the overexpression of Sirt6 on the protein expression levels of Bcl-2, Bax and Sirt6 in the Transwell co-culture system. (E) Relative protein levels are presented as bar diagrams. GAPDH was used as an internal control. Data are presented as the means \pm SD from 3 independent experiments ( $\left({ }^{*}<0.05\right.$ and ${ }^{* *} \mathrm{P}<0.01$, compared with the $\mathrm{NM}+\mathrm{HG}+\mathrm{Con}$ group; ${ }^{\wedge} \mathrm{P}<0.01$, compared with the $\mathrm{HG}+\mathrm{NC}+\mathrm{Con}$ group; ${ }^{\# \#} \mathrm{P}<0.01$, compared with the M-Sirt6 group; ${ }^{\& \&} \mathrm{P}<0.01$, compared with the HG + M-Sirt6 group).

the possible mechanisms responsible for the protective effects of Sirt6 overexpression against podocyte injury. We found that the overexpression of Sirt6 in macrophages inhibited the expression of the M1 marker, CD86 $(\mathrm{P}<0.01$; Fig. 7A, D and E), and promoted that of CD206 ( $<<0.01$; Fig. 7B, D and E), which is a marker of the M2 phenotype expression, in the high-glucose environment at both the mRNA and protein level. When Sirt6 overexpression was induced in macrophages by transfection with Sirt6 overexpression plasmid, the results revealed an increased mRNA and protein expression of Sirt6 in the mimicked diabetic internal environment $(\mathrm{P}<0.01$; Fig. $7 \mathrm{C}-\mathrm{E})$. On the basis of the above-mentioned results, we hypothesized that the overexpression of Sirt6 in macrophages promoted macrophage M2 transformation, thus inhibiting podocyte apoptosis under high-glucose conditions.

\section{Discussion}

In this study, we used streptozocin to induce diabetes in rats as previously described (25-28). The inflammatory process and potocyte injury in the rats with DN were subsequently analyzed. Podocytes play an important role in maintaining glomerular structural integrity and filtration function. Podocytes are characterized by being able to express specific functional protein molecules, such as Nephrin, 

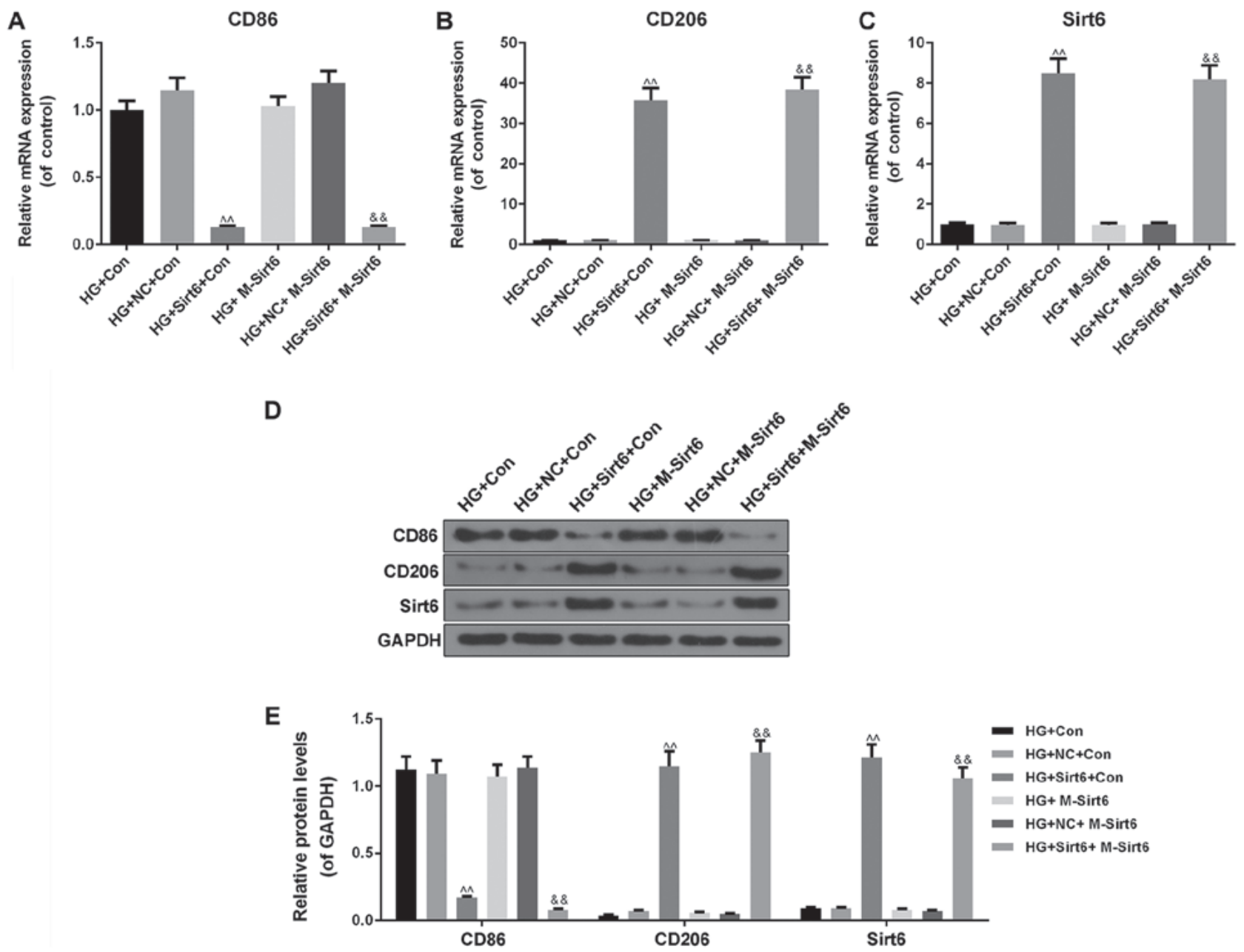

Figure 7. Overexpression of Sirt6 in macrophages regulates the expression of markers of macrophages and Sirt6 under high-glucose conditions. The diabetic internal environment was mimicked by a Transwell co-culture system under high-glucose conditions to co-culture the macrophages and podocytes. The cells in this experiment were divided into 8 groups as follows: i) The $\mathrm{NM}+\mathrm{HG}+$ Con group (control; $50 \mathrm{mmol} / \mathrm{l}$ of high-glucose medium in the upper chamber, and podocytes with high-glucose medium in the lower chamber); ii) the $\mathrm{HG}+\mathrm{Con}$ group (macrophages with high-glucose medium in the upper chamber, and podocyte with high-glucose medium in the lower chamber); iii) the $\mathrm{HG}+\mathrm{NC}+\mathrm{Con}$ group (macrophages transfected with NC plasmid with high-glucose medium in the upper chamber, and podocytes with high-glucose medium in the lower chamber); iv) the HG + Sirt6 + Con group (macrophages transfected with Sirt6 overexpression plasmid with high-glucose medium in the upper chamber, and podocytes with high-glucose medium in the lower chamber); v) the M-Sirt6 group (high-glucose medium in the upper chamber, and podocytes transfected with Sirt6 overexpression plasmid with high-glucose medium in the lower chamber); vi) the HG + M-Sirt6 group (macrophages with high-glucose medium in the upper chamber, and podocytes transfected with Sirt6 overexpression plasmid with high-glucose medium in the lower chamber); vii) the HG + NC + M-Sirt6 group (macrophages transfected with NC plasmid with high-glucose medium in the upper chamber, and podocytes transfected with Sirt6 overexpression plasmid with high-glucose medium in the lower chamber); and viii) the HG + Sirt6 + M-Sirt6 (macrophages transfected with Sirt6 overexpression plasmid with high-glucose medium in the upper chamber, and podocytes transfected with Sirt6 overexpression plasmid with high-glucose medium in the lower chamber). RT-qPCR was performed to examine the effects of the overexpression of Sirt6 on the mRNA expression levels of (A) CD86, (B) CD206, and (C) Sirt6 in the Transwell co-culture system. (D) Western blot analysis was performed to examine the effects of the overexpression of Sirt6 on the protein expression levels of CD86, CD206 and Sirt6 in the Transwell co-culture system. (E) Relative protein levels are presented as bar diagrams. GAPDH was used as an internal control. Data are presented as the means \pm SD from 3 independent experiments $\left({ }^{\wedge} \mathrm{P}<0.01\right.$, compared with the HG + Con group; ${ }^{\&} \mathrm{P}<0.01$, compared with the HG + M-Sirt6 group).

Podocin, Podocalyxin and Desmin (29-33). Podocyte injury is considered to be the leading cause of proteinuria and glomerular disease $(34,35)$. In this study, in the tissues of rats with DN, we found that Nephrin protein expression decreased and Desmin protein expression increased. These are typical podocyte injury markers that allow Nephrin gene mutation to lead to proteinuria production and congenital nephrotic syndrome. In addition, a high expression of Desmin and its phenotypic transformation causes the re-arrangement of the podocyte cytoskeleton $(36,37)$. Previously, Bohle et al reported that a large number of inflammatory cells, which either were macrophages, or were lymphocytes, were found in renal tissues of 488 patients with DN by immunostaining; it was thus hypothesized that the pathogenesis of DN was related to tubulointerstitial immune inflammation (38). An increasing number of studies have confirmed that macrophage aggregation is a predictor of renal dysfunction in patients with DN $(9,23,39-41)$. In this study, we detected the degree of macrophage infiltration and associated inflammatory factor expression in rats with DN. Some studies have found that symbolic molecules of M1 macrophages include TNF- $\alpha$, IL-6, monocyte chemoattractant protein-1 (MCP-1) and iNOS, while representative $\mathrm{M} 2$ macrophages molecules include IL-10, CD206, CD163 and chemokine (C-C motif) ligand 18 
(CCL18; also known as AMAC1) $(42,43)$. The findings of this study demonstrated that CD86 expression was significantly upregulated under high-glucose conditions and that of CD206 exhibited an opposite trend. Additionally, TNF- $\alpha$ and iNOS mRNA expression levels increased and the mRNA expression levels of IL-4 and IL-10 decreased under high-glucose conditions. Recently, Assadi-Porter et al reported the effects of 3-iodothyronamine (T1AM) on obesity and their results also demonstrated that these effects were co-regulated by Sirt6 and Sirt4; in detail, Sirt6 regulated glycolysis, triglyceride synthesis and fat metabolism, while Sirt4 had an opposite effect, negatively regulating fatty acid oxidation (44). The results of their study were similar to those of this study in that the expression of Sirt6 was significantly decreased in the tissues of rats with DN. Furthermore, in future studies, we aim to examine the co-regulation of Sirt 6 and Sirt 4 in diabetes-induced renal injury.

In this study, macrophages and podocytes were respectively used to conduct in vitro experiments with a series of detections. The results revealed that glucose promoted macrophage M1 transformation and podocyte apoptosis in a dose-dependent manner, and high glucose decreased Sirt6 expression in rats with DN.

Increasing evidence indicates that Sirtuins, particularly Sirt1, Sirt3 and Sirt6, are related to kidney disease (45-47). Several studies have demonstrated that the expression of Sirt1 in DN decreases, and that the increased Sirtl expression can attenuate renal injury in DN (48-51). Caton et al reported that the decreased expression of Sirt 3 resulted in an impaired function of $\beta$-cells in type 2 diabetes mellitus (52). Sirt3, as a protective agent of acute tissue injury, has also been shown to play an important role in acute renal injury model (53). Sirt6 is considered to be related to the occurrence and development of chronic diseases, including diabetes, and such an effect is realized by its role in DNA damage (54-56). Therefore, in this study, the overexpression of Sirt6 was successfully indcued in macrophages and podocytes, respectively, and we found that the overexpression of Sirt6 promoted macrophage M2 transformation. It is unusual that a lack of Sirt6 would increase the inflammatory response in tissues. For example, a lack of Sirt6 in adipose tissue has been shown to increased macrophage infiltration and adipose tissue inflammation and to promote HFD-induced insulin resistance $(57,58)$. In addition, its deficiency causes liver inflammation and fibrosis in mouse immune cells (59). However, the effects of Sirt4 on related inflammation, insulin resistance and oxidative metabolism are not consistent with those of Sirt6. For example, Sirt4 modulates oxidative metabolism and directly leads to endothelial dysfunction, and is related to coronary artery disease (60). Huynh et al reported that the loss of Sirt4 elevated glucose- and leucine-stimulated insulin levels in vivo, which were dependent upon a C57BL/6NJ genetic background, and enhanced age-induced insulin resistance (61). This is a very interesting research prospect, which is worthy of further study.

Furthermore, in this study, we mimicked the diabetic internal environment by a Transwell co-culture system under high-glucose conditions to co-culture macrophages and podocytes. We found that the overexpression of Sirt6 in macrophages protected podocytes from high-glucose-induced injury, whereas the overexpression of Sirt6 in podocytes exerted no protective effect itself against injury induced by high glucose. In addition, we found that the possible mechanism responsible for the protective effects of Sirt6 overexpression in macrophages against podocyte injury involved the promotion of the macrophage M2 transformation. Such a result is in line with the findings from the study by Sun et al, who reported that by inducing M2 macrophage differentiation, pentraxin-3 alleviated kidney damage in DN (23). Zhang et al also suggested that the inhibition of M1 macrophage activation and the promotion of M2 macrophage transformation prevented podocyte injury (62).

Taken together, the findings of this study suggested that Sirt6 protected against podocytes from injury in a mimicked diabetic kidney microenvironment by activating M2 macrophages. Our findings also indicated that Sirt6 functioned as an immune response regulatory factor in renal inflammatory injury induced by streptozotocin administration in rats. It would be more convincing to provide more kidney function indicators [blood urea nitrogen (BUN) and serum creatinine (SCr)] and a more general method for modeling DN in animals. These are the limitations of our study and we aim to perform a more detailed and in-depth analysis in the future.

\section{Acknowledgements}

Not applicable.

\section{Funding}

This study was supported by the Hangzhou Science and Technology Commission 'the Warehousing project of Hangzhou Agricultural and Social Development Research Project Library' (164783); the Zhejiang Provincial Chinese Medicine Administration 'Zhejiang Traditional Chinese Medicine Research Fund Project' (2016ZA155); and the Zhejiang Provincial Health and Family Planning Commission 'Zhejiang Medical and Health Research Fund Project' (2017KY129).

\section{Availability of data and materials}

The analyzed data sets generated during the study are available from the corresponding author on reasonable request.

\section{Authors' contributions}

LJ made substantial contributions to the conception and design of the study. HW and WZ were involved in data acquisition, data analysis and interpretation. YC and LH were involved in the cell experiments and the drafting of the article or critically revising it for important intellectual content. JW and YL were involved in the animal experiments. All authors are accountable for data integrity, and have read and approved the final manuscript.

\section{Ethics approval and consent to participate}

The animal experiments were performed according to the Guide for the Care and Use of Laboratory Animals and 
approved by the First People's Hospital of Yuhang District, Hangzhou Animal Ethics Committee (Hangzhou, China).

\section{Patient consent for publication}

Not applicable.

\section{Competing interests}

The authors declare that they have no competing interests.

\section{References}

1. Gallagher H and Suckling RJ: Diabetic nephropathy: Where are we on the journey from pathophysiology to treatment? Diabetes Obes Metab 18: 641-647,2016.

2. Dronavalli S, Duka I and Bakris GL: The pathogenesis of diabetic nephropathy. Nat Clin Pract Endocrinol Metab 4: 444-452, 2008.

3. Ma R, Liu L, Jiang W, Yu Y and Song H: FK506 ameliorates podocyte injury in type 2 diabetic nephropathy by down-regulating TRPC6 and NFAT expression. Int J Clin Exp Pathol 8: 14063-14074, 2015.

4. Eremina V, Baelde HJ and Quaggin SE: Role of the VEGF--a signaling pathway in the glomerulus: Evidence for crosstalk between components of the glomerular filtration barrier. Nephron, Physiol 106: 32-37, 2007.

5. Mundel P and Kriz W: Structure and function of podocytes: An update. Anat Embryol (Berl) 192: 385-397, 1995.

6. Mathieson PW: What has the immune system got against the glomerular podocyte? Clin Exp Immunol 134: 1-5, 2003.

7. Gordon S and Taylor PR: Monocyte and macrophage heterogeneity. Nat Rev Immunol 5: 953-964, 2005.

8. Chow F, Ozols E, Nikolic-Paterson DJ, Atkins RC and Tesch GH: Macrophages in mouse type 2 diabetic nephropathy: Correlation with diabetic state and progressive renal injury. Kidney Int 65: 116-128, 2004.

9. Nguyen D, Ping F, Mu W, Hill P, Atkins RC and Chadban SJ: Macrophage accumulation in human progressive diabetic nephropathy. Nephrology (Carlton) 11: 226-231, 2006.

10. Tesch GH: Macrophages and diabetic nephropathy. Semin Nephrol 30: 290-301, 2010.

11. Minton K: Macrophages: A transcription factor to call their own. Nat Rev Immunol 11: 74, 2011.

12. Hofkens W, Storm G, Berg WVD and Lent PV: Inhibition of M1 macrophage activation in favour of M2 differentiation by liposomal targeting of glucocorticoids to the synovial lining during experimental arthritis. Ann Rheum Dis 70 (Suppl 2): 70, 2011.

13. Gordon S: Alternative activation of macrophages. Nat Rev Immunol 3: 23-35, 2003.

14. Gertler AA and Cohen HY: SIRT6, a protein with many faces Biogerontology 14: 629-639, 2013.

15. Tennen RI, Berber E and Chua KF: Functional dissection of SIRT6: Identification of domains that regulate histone deacetylase activity and chromatin localization. Mech Ageing Dev 131: 185-192, 2010.

16. Tennen RI, Bua DJ, Wright WE and Chua KF: SIRT6 is required for maintenance of telomere position effect in human cells. Nat Commun 2: 433, 2011.

17. Beauharnois JM, Bolívar BE and Welch JT: Sirtuin 6: A review of biological effects and potential therapeutic properties. Mol Biosyst 9: 1789-1806, 2013.

18. Michishita E, McCord RA, Berber E, Kioi M, Padilla-Nash H, Damian M, Cheung P, Kusumoto R, Kawahara TL, Barrett JC, et al: SIRT6 is a histone $\mathrm{H} 3$ lysine 9 deacetylase that modulates telomeric chromatin. Nature 452: 492-496, 2008.

19. Yuan J, Pu M, Zhang Z and Lou Z: Histone H3-K56 acetylation is important for genomic stability in mammals. Cell Cycle 8: 1747-1753, 2009.

20. Mostoslavsky R, Chua KF, Lombard DB, Pang WW, Fischer MR, Gellon L, Liu P, Mostoslavsky G, Franco S, Murphy MM, et al: Genomic instability and aging-like phenotype in the absence of mammalian SIRT6. Cell 124: 315-329, 2006.

21. Zhong L, D'Urso A, Toiber D, Sebastian C, Henry RE, Vadysirisack DD, Guimaraes A, Marinelli B, Wikstrom JD, Nir T, et al: The histone deacetylase Sirt6 regulates glucose homeostasis via Hif1alpha. Cell 140: 280-293, 2010.
22. Wang X, Liu J, Zhen J, Zhang C, Wan Q, Liu G, Wei X, Zhang Y, Wang Z, Han H, et al: Histone deacetylase 4 selectively contributes to podocyte injury in diabetic nephropathy. Kidney Int 86: 712-725, 2014.

23. Sun H, Tian J, Xian W, Xie T and Yang X: Pentraxin-3 attenuates renal damage in diabetic nephropathy by promoting M2 macrophage differentiation. Inflammation 38: 1739-1747, 2015.

24. Livak KJ and Schmittgen TD: Analysis of relative gene expression data using real-time quantitative PCR and the 2(-Delta Delta C(T)) Method. Methods 25: 402-408, 2001.

25. Zanotto C, Hansen F, Galland F, Batassini C, Federhen BC, da Silva VF, Leite MC, Nardin P and Gonçalves CA: Glutamatergic alterations in STZ-induced diabetic rats are reversed by Exendin-4. Mol Neurobiol: Aug 25, 2018 (Epub ahead of print). doi: 10.1007/s12035-018-1320-5. 2018.

26. Nunes KP, de Oliveira AA, Szasz T, Biancardi VC and Webb RC: Blockade of toll-like receptor 4 attenuates erectile dysfunction in diabetic rats. J Sex Med 15: 1235-1245, 2018.

27. Huang YC, Wu BH, Chu YL, Chang WC and Wu MC: Effects of tempeh fermentation with Lactobacillus plantarum and Rhizopus oligosporus on streptozotocin-induced type II diabetes mellitus in rats. Nutrients 10: 10, 2018.

28. Wu M, Yang Y, Wang M, Zeng F, Li Q, Liu W, Guo S, He M, Wang Y, Huang J, et al: Exogenous pancreatic kallikrein improves diabetic cardiomyopathy in streptozotocin-induced diabetes. Front Pharmacol 9: 855, 2018.

29. Inoki K, Mori H, Wang J, Suzuki T, Hong S, Yoshida S, Blattner SM, Ikenoue T, Rüegg MA, Hall MN, et al: mTORC1 activation in podocytes is a critical step in the development of diabetic nephropathy in mice. J Clin Invest 121: 2181-2196, 2011.

30. Wang W, Wang Y, Long J, Wang J, Haudek SB, Overbeek P, Chang BH, Schumacker PT and Danesh FR: Mitochondrial fission triggered by hyperglycemia is mediated by ROCK1 activation in podocytes and endothelial cells. Cell Metab 15: 186-200, 2012.

31. Casalena G, Krick S, Daehn I, Yu L, Ju W, Shi S, Tsai SY, D'Agati V, Lindenmeyer M, Cohen CD, et al: Mpv17 in mitochondria protects podocytes against mitochondrial dysfunction and apoptosis in vivo and in vitro. Am J Physiol Renal Physiol 306: F1372-F1380, 2014.

32. Bechtel W, Helmstädter M, Balica J, Hartleben B, Kiefer B, Hrnjic F, Schell C, Kretz O, Liu S, Geist F, et al: Vps34 deficiency reveals the importance of endocytosis for podocyte homeostasis. J Am Soc Nephrol 24: 727-743, 2013.

33. Liu N, Xu L, Shi Y and Zhuang S: Podocyte autophagy: A potential therapeutic target to prevent the progression of diabetic nephropathy. J Diabetes Res 2017: 3560238, 2017.

34. Inoue R, Asanuma K, Seki T, Nagase M and Osafune K: New therapeutic insights for chronic kidney disease provided by podocytology. Nihon Yakurigaku Zasshi 143: 27-33, 2014 (In Japanese).

35. Lennon R, Randles MJ and Humphries MJ: The importance of podocyte adhesion for a healthy glomerulus. Front Endocrinol (Lausanne) 5: 160, 2014

36. Kestilä M, Lenkkeri U, Männikkö M, Lamerdin J, McCready 6P, Putaala H, Ruotsalainen V, Morita T, Nissinen M, Herva R, et al: Positionally cloned gene for a novel glomerular protein - nephrin is mutated in congenital nephrotic syndrome. Mol Cell 1: 575-582, 1998.

37. Wang Z, Liu J and Sun W: Effects of asiaticoside on levels of podocyte cytoskeletal proteins and renal slit diaphragm proteins in adriamycin-induced rat nephropathy. Life Sci 93: 352-358, 2013.

38. Bohle A, Wehrmann M, Bogenschütz O, Batz C, Müller CA and Müller GA: The pathogenesis of chronic renal failure in diabetic nephropathy. Investigation of 488 cases of diabetic glomerulosclerosis. Pathol Res Pract 187: 251-259, 1991.

39. Furuta T, Saito T, Ootaka T, Soma J, Obara K, Abe K and Yoshinaga K: The role of macrophages in diabetic glomerulosclerosis. Am J Kidney Dis 21: 480-485, 1993.

40. Yonemoto S, Machiguchi T, Nomura K, Minakata T, Nanno M and Yoshida $\mathrm{H}$ : Correlations of tissue macrophages and cytoskeletal protein expression with renal fibrosis in patients with diabetes mellitus. Clin Exp Nephrol 10: 186-192, 2006.

41. Guo Y, Song Z, Zhou M, Yang Y, Zhao Y, Liu B and Zhang X: Infiltrating macrophages in diabetic nephropathy promote podocytes apoptosis via TNF- $\alpha$-ROS-p38MAPK pathway. Oncotarget 8: 53276-53287, 2017. 
42. Liu H, Dong H, Jiang L, Li Z and Ma X: Bleomycin inhibits proliferation and induces apoptosis in TPC-1 cells through reversing M2-macrophages polarization. Oncol Lett 16: 3858-3866, 2018.

43. Heusinkveld M, de Vos van Steenwijk PJ, Goedemans R, Ramwadhdoebe TH, Gorter A, Welters MJ, van Hall T and van der Burg SH: M2 macrophages induced by prostaglandin E2 and IL-6 from cervical carcinoma are switched to activated M1 macrophages by CD4+ Th1 cells. J Immunol 187: 1157-1165, 2011.

44. Assadi-Porter FM, Reiland H, Sabatini M, Lorenzini L, Carnicelli V, Rogowski M, Selen Alpergin ES, Tonelli M, Ghelardoni S, Saba A, et al: Metabolic reprogramming by 3-iodothyronamine (T1AM): A new perspective to reverse obesity through co-regulation of sirtuin 4 and 6 expression. Int J Mol Sci 19: 19, 2018.

45. Aditya R, Kiran AR, Varma DS, Vemuri R and Gundamaraju R: A review on SIRtuins in diabetes. Curr Pharm Des 23: 2299-2307, 2017.

46. Feng J, Lu C, Dai Q, Sheng J and Xu M: SIRT3 facilitates amniotic fluid stem cells to repair diabetic nephropathy through protecting mitochondrial homeostasis by modulation of mitophagy. Cell Physiol Biochem 46: 1508-1524, 2018.

47. Huynh FK, Hershberger KA and Hirschey MD: Targeting sirtuins for the treatment of diabetes. Diabetes Manag (Lond) 3 : 245-257, 2013

48. Kitada M, Takeda A, Nagai T, Ito H, Kanasaki K and Koya D: Dietary restriction ameliorates diabetic nephropathy through anti-inflammatory effects and regulation of the autophagy via restoration of Sirt1 in diabetic Wistar fatty (fa/fa) rats: A model of type 2 diabetes. Exp Diabetes Res 2011: 908185, 2011.

49. Li C, Cai F, Yang Y, Zhao X, Wang C, Li J, Jia Y, Tang J and Liu Q: Tetrahydroxystilbene glucoside ameliorates diabetic nephropathy in rats: Involvement of SIRT1 and TGF- $\beta 1$ pathway. Eur J Pharmacol 649: 382-389, 2010.

50. Shang G, Gao P, Zhao Z, Chen Q, Jiang T, Zhang N and Li H: 3,5-Diiodo-1-thyronine ameliorates diabetic nephropathy in streptozotocin-induced diabetic rats. Biochim Biophys Acta 1832: 674-684, 2013.

51. Wang X, Meng L, Zhao L, Wang Z, Liu H, Liu G and Guan G: Resveratrol ameliorates hyperglycemia-induced renal tubular oxidative stress damage via modulating the SIRT1/FOXO3a pathway. Diabetes Res Clin Pract 126: 172-181, 2017.

52. Caton PW, Richardson SJ, Kieswich J, Bugliani M, Holland ML, Marchetti P, Morgan NG, Yaqoob MM, Holness MJ and Sugden MC: Sirtuin 3 regulates mouse pancreatic beta cell function and is suppressed in pancreatic islets isolated from human type 2 diabetic patients. Diabetologia 56: 1068-1077, 2013.
53. Morigi M, Perico L, Rota C, Longaretti L, Conti S, Rottoli D, Novelli R, Remuzzi G and Benigni A: Sirtuin 3-dependent mitochondrial dynamic improvements protect against acute kidney injury. J Clin Invest 125: 715-726, 2015.

54. Blasiak J, Arabski M, Krupa R, Wozniak K, Zadrozny M, Kasznicki J, Zurawska M and Drzewoski J: DNA damage and repair in type 2 diabetes mellitus. Mutat Res 554: 297-304, 2004.

55. Grindel A, Guggenberger B, Eichberger L, Pöppelmeyer C, Gschaider M, Tosevska A, Mare G, Briskey D, Brath H and Wagner KH: Oxidative stress, DNA damage and DNA repair in female patients with diabetes mellitus type 2. PLoS One 11: e0162082, 2016.

56. Kuang J, Chen L, Tang Q, Zhang J, Li Y and He J: The role of Sirt6 in obesity and diabetes. Front Physiol 9: 135, 2018.

57. Kuang J, Zhang Y, Liu Q, Shen J, Pu S, Cheng S, Chen L, Li H, Wu T, Li R, et al: Fat-specific Sirt6 ablation sensitizes mice to high-fat diet-induced obesity and insulin resistance by inhibiting lipolysis. Diabetes 66: 1159-1171, 2017.

58. Xiong X, Zhang C, Zhang Y, Fan R, Qian X and Dong XC: Fabp4-Cre-mediated Sirt6 deletion impairs adipose tissue function and metabolic homeostasis in mice. J Endocrinol 233 307-314, 2017.

59. Xiao C, Wang RH, Lahusen TJ, Park O, Bertola A, Maruyama T, Reynolds D, Chen Q, Xu X, Young HA, et al: Progression of chronic liver inflammation and fibrosis driven by activation of c-JUN signaling in Sirt6 mutant mice. J Biol Chem 287: 41903-41913, 2012

60. Tarantino G, Finelli C, Scopacasa F, Pasanisi F, Contaldo F, Capone D and Savastano S: Circulating levels of sirtuin 4, a potential marker of oxidative metabolism, related to coronary artery disease in obese patients suffering from NAFLD, with normal or slightly increased liver enzymes. Oxid Med Cell Longev 2014: 920676, 2014.

61. Huynh FK, Hu X, Lin Z, Johnson JD and Hirschey MD: Loss of sirtuin 4 leads to elevated glucose- and leucine-stimulated insulin levels and accelerated age-induced insulin resistance in multiple murine genetic backgrounds. J Inherit Metab Dis 41: 59-72, 2018

62. Zhang XL, Guo YF, Song ZX and Zhou M: Vitamin D prevents podocyte injury via regulation of macrophage M1/M2 phenotype in diabetic nephropathy rats. Endocrinology 155: 4939-4950, 2014. International (CC BY-NC-ND 4.0) License. 